\title{
Charge Transport with Single Molecules - An Electrochemical Approach
}

\author{
Chen Li, Artem Mishchenko, llya Pobelov, and Thomas Wandlowski ${ }^{\star}$
}

\begin{abstract}
After an introduction and brief review of charge transport in nanoscale molecular systems we report on experimental studies in gold | (single) molecule | gold junctions at solid | liquid interfaces employing a scanning tunneling microscopy (STM)-based 'break junction' technique. We demonstrate attempts in developing basic relationships between molecular structure, conductance properties and nanoscale electrochemical concepts based on four case studies from our own work. In experiments with $\alpha, \omega$-alkanedithiol and biphenyldithiol molecular junctions we address the role of sulfur-gold couplings and molecular conformation, such as gauche defects in the alkyl chains and the torsion angle between two phenyl rings. Combination with quantum chemistry calculations enabled a detailed molecular-level understanding of the electronic structure and transport characteristics of both systems. Employing the concept of 'electrolyte gating' with redox-active molecules, such as thiol-terminated derivatives of viologens (HS-6V6-SH or (HS-6V6)) we demonstrate the construction of symmetric and asymmetric active molecular junctions with transistor- or diode-like behavior upon polarization in an electrochemical environment. The experimental data could be represented quantitatively by the Kutznetsov/Ulstrup model assuming a two-step electron transfer with partial vibration relaxation. Finally, we show that surface-immobilized gold nanoparticles with a diameter of $(2.4 \pm 0.5) \mathrm{nm}$ exhibit features of locally addressable multi-state electronic switching upon electrolyte gating, which appears to be reminiscent of a sequential charging through several 'oxidation/reduction states'.
\end{abstract}

Keywords: Electrolyte gating · Quantized cluster charging · Single molecule electron transport · STM-based break junction

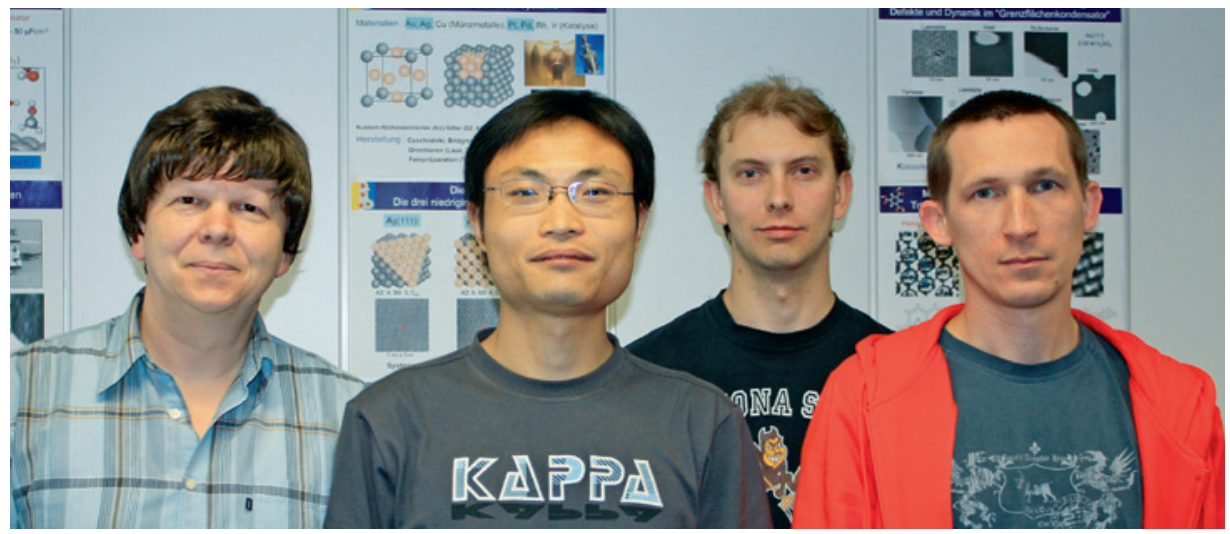

Thomas Wandlowski, Chen Li, llya Pobelov and Artem Mishchenko.

Chen $\mathbf{L i}$ studied chemistry at Xiamen University, PR China. He graduated with a master thesis on synthesis and characterization of one-dimensional semiconductor materials under Professor Y. Yang. He

${ }^{*}$ Correspondence: Prof. Dr. T. Wandlowsk University of Bern

Department of Chemistry and Biochemistry

Freiestrasse 3

$\mathrm{CH}-3012$ Bern

Tel.: +41316315384

Fax: + 41316313994

E-mail: thomas.wandlowski@dcb.unibe.ch joined the Surface Electrochemistry group of T. Wandlowski at the Research Center Jülich as a PhD student in 2006. Chen Li's dissertation involved electron transport studies of single molecules with a specific focus on electrochemical scanning tunneling microscopy. He works currently as a postdoc in the Wandlowski group in Bern.

Artem Mishchenko studied biochemistry at Kazan University. After graduation with honors he accepted a junior assistant fellowship in Physical Chemistry at the Chemical Institute of Kazan University in 2001. During this time he investigated sorption processes of organic compounds at various substrates. Artem Mishchenko joined the Wandlowski group in 2007 as PhD student.
His current research is focused on structure aspects of single molecule conductance in metal | molecule | metal assemblies. He is particularly interested in developing and exploring novel instrumental approaches.

Ilya Pobelov is a graduate of Moscow State University. He completed his MS thesis work entitled 'Electroreduction of Pt(II)-chloroaquocomplexes' under supervision of Prof. G. Tsirlina in 2002. After a one-year junior assistantship at the Department of Electrochemistry at Moscow State University he joined the group of T. Wandlowski as a PhD student and DAAD fellow in 2003. His $\mathrm{PhD}$ project involved experimental and theoretical studies on electron transport in electrochemical system. After graduation with honors at the RWTH in Aachen in 2008, he started his term as research associate in the Bern group. His current research interests are particularly geared towards the development and applications of novel scanning probe and spectroscopic techniques in nanoscale electrochemical systems.

Thomas Wandlowski studied chemistry in Halle, Germany. After graduation in 1983 he joined the group of Prof. E. Kretschmer as $\mathrm{PhD}$ student with an experimental topic on electrocapillary measurements at mercury/electrolyte interface. This work was extended towards ions transfer and adsorption studies at liquid/liquid interfaces dur- 
ing a postdoctoral stay with Prof. Z. Samec at the J. J. Heyrovsky Institute in Prague. After successfully completing his habilitation on two-dimensional phase transitions in organic adlayers at electrochemical interfaces in Halle in 1990, T. Wandlowski accepted a research assistant professorship at Georgetown University. He returned to Europe with a Heisenberg fellowship of the DFG in 1994, and started building up his own group at Ulm University. After eight years as head of the electrochemistry group at the Institute of Surfaces and Interfaces in the Research Center Jülich and lecturing at the RWTH Aachen T. Wandlowski moved together with his Jülich group as a full Professor of Physical Chemistry and Electrochemistry to the University of Bern. His research interests cover a wide range of molecular-scale processes and phenomena at well-defined electrified interfaces combining classical macroscopic electrochemical techniques with in situ structure-sensitive approaches and methods.

\section{Introduction - Charge Transport in Nanoscale Molecular Systems}

The idea of building an electronic device using individual molecules was first proposed by Aviram and Ratner. ${ }^{[1]}$ The molecular approach bears several opportunities: i) custom-design of nanoscale molecular units, ii) self-organization and recognition properties of molecular building blocks, iii) implementation of localized functions such as rectification, switching or amplification. ${ }^{[2-9]}$ The ability to measure and to control charge transport across nanometer-scale metal | (single) molecule | metal junctions is of considerable fundamental interest and represents a key step towards the development of a molecularbased electronics. Various experimental approaches have been employed to study molecular junctions in two- and threeterminal configurations. These include scanning probe microscopies (STM, ${ }^{[10-13]}$ CP-AFM,[14-16] STS[17-20]), crossed wire[21] and nanoparticle junctions, ${ }^{[22,23]}$ mechani$\mathrm{cal}^{[24-28]}$ and electromigration break junctions, ${ }^{[29,30]}$ nanopores ${ }^{[31]}$ and mercury drop electrodes. ${ }^{[32]}$ Most experiments refer to $e x$ situ conditions, i.e. in air or vacuum, and at cryogenic temperatures. Approaches in condensed media, and in particular in an electrochemical environment (liquid electrolytes or solid-state ionic conductors), offer unique opportunities to explore charge transport across single molecules employing the concept of 'electrolyte gating'.

Molecular wires studied to date can be divided in two classes: i) $\sigma$-bonded aliphatic molecules and ii) $\pi$-bonded aromatic molecules. ${ }^{[33,34]} \alpha, \omega$-alkanedithi-

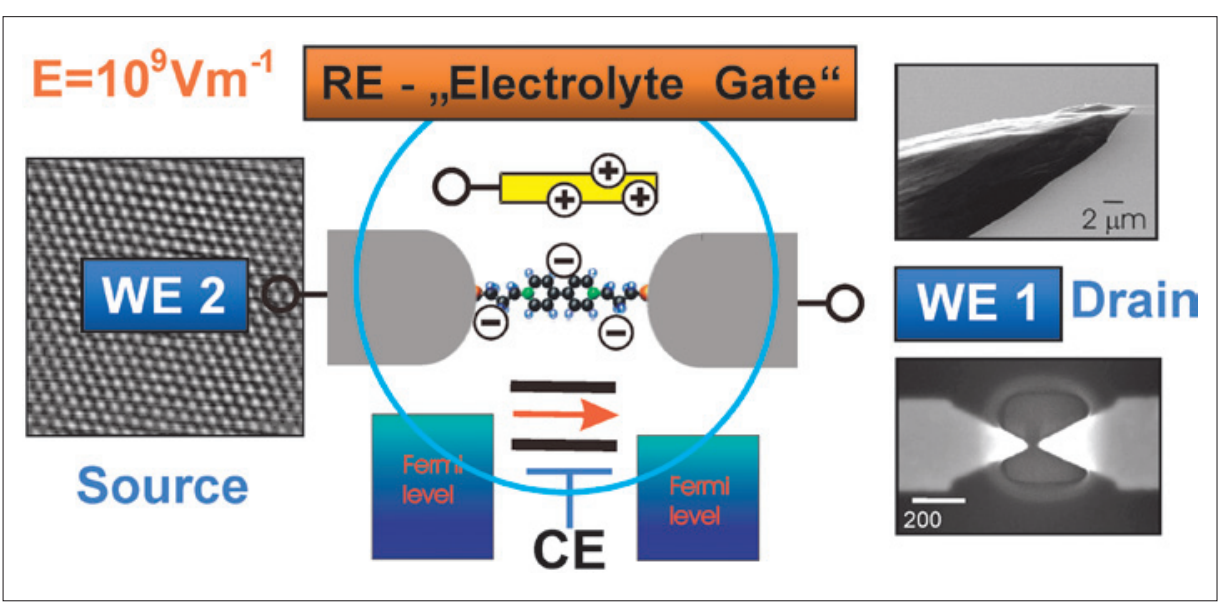

Fig. 1. Principle of 'electrolyte gating'. Tuning of the Fermi levels of WE 1 and WE 2 relative to the molecular levels enables measuring of current (i) - voltage $(E)$ characteristics i vs $\left(E_{W E 1}-E_{W E 2}\right)$ at fixed $E_{W E 1}$ or $E_{W E 2}$, i vs. $E_{W E 1}$ or $E_{W E 2}$ at fixed bias $E_{\text {bias }}=\left(E_{W E 1}-E_{W E 2}\right)$ as well as barrier height profiles $i$ vs. distance of tailored molecular junctions in a vertical SPM-configuration respective horizontal nanoelectrode assembly.

ols ([14,15,17,35] and literature cited therein), oligophenylene vinylene (OPV) and oligophenylene ethynylene (OPE) rods $([10,16,36]$ and literature cited therein) attached to gold electrodes or nanocrystals via thiol linkers are prominent examples. Except of a systematic study with aliphatic and aromatic diamines of the Columbia group ref. [32] and literature cited therein), only few reports on other anchoring groups to establish electronic coupling between molecular wires and metal contacts were published. These include carboxylic acid groups, ${ }^{[38,39]}$ isocyanide, ${ }^{[40,41]}$ nitrile ${ }^{[40]}$ and selenol.[42] First studies with substituted $\alpha, \omega$-alkane derivatives by Huggins ${ }^{[43]}$ and Chen et al., ${ }^{[38]}$ and aromatic molecular wires by Venkataraman et al., [37] Haiss et $a l .{ }^{[44]}$ and us ${ }^{[45,46]}$ at solid liquid interfaces illustrated the unique potential of chemical control, e.g. the effect of electron-donating respective -withdrawing substituents, molecular conformation or anchoring groups on the conductance signature of single molecular junctions. However, there is still a considerable lack in understanding basic relationships between molecular structure and conductance properties. ${ }^{[5-9]}$

Several groups explored 'active' solid state metal | molecule | metal junctions, for instance with redox centers under ex situ (ambient) and UHV (cryogenic) conditions. They discovered phenomena such as negative differential resistance (NDR), ${ }^{[31]}$ rectification, $[7,47,48]$ amplification, ${ }^{[28,29]}$ switching and memory effects. ${ }^{[8,49-51]}$ Examples of functional molecules used in these studies are 2'-amino-4,4'-di(ethynylphenyl)5 '-nitro-1-benzene thiolate, ${ }^{[31]}$ metal organic $\mathrm{Co}^{2+}$-terpyridine complexes ${ }^{[29]}$ or a $\left[\mathrm{Co}_{4} \mathrm{~L}_{4}\right]^{8+}-[2 \times 2]$ grid type complex. ${ }^{[30]}$ The experimental investigations are currently accompanied by powerful theoretical and computational approaches to interpret molecular signatures in these transport junctions. ${ }^{5]}$ New challenges and unique opportunities emerge when adapting these studies to nanoscale systems in an electrochemical environment. ${ }^{[52,53]}$

\section{The Electrochemical Approach}

The addressing of nanoelectronic assemblies metal | 'molecule' (nanocluster) | metal with device-like functions, such as rectifiers, switches, transistors requires a source and a drain and one or more localized electronic levels. ${ }^{[5]}$ The roles of source and drain (both as working electrodes WE1 and WE2) may be represented by the tip of a STM combined with an appropriate substrate or, alternatively, a pair of nanoelectrodes (Fig. 1).

Working in an electrochemical environment such as an electrified solid | liquid interface has the advantage that two potential differences can be controlled individually: the bias voltage between the two working electrodes (WE1 and WE2) and the potential drop between each individual working electrode and the reference electrode (RE). The latter acts as an 'electrochemical gate' and modulates the tunneling current between source (WE2) and drain (WE1). ${ }^{[52,53]}$ The idea of an 'electrochemical gate' to control charge transport in molecular electronics was introduced by Wrighton, [54] Meulenkamp, ${ }^{[55]}$ Schönenberger,[56] and McEuen, [57] and further developed by Tao, ${ }^{[18,58,59]}$ Ulstrup et al., ${ }^{[52,53,60]}$ Lindsay, ${ }^{[61]}$ Haiss et al., ${ }^{[19]}$ Vanmaekelbergh, ${ }^{[62]}$ and us. ${ }^{[20,63]}$ The effective gate | molecule distance is determined by the double layer thickness at the electrode | electrolyte interface, which is typically of the size of a few solvated ions. The 'electrolyte gating' ensures a strong coupling due to low con- 


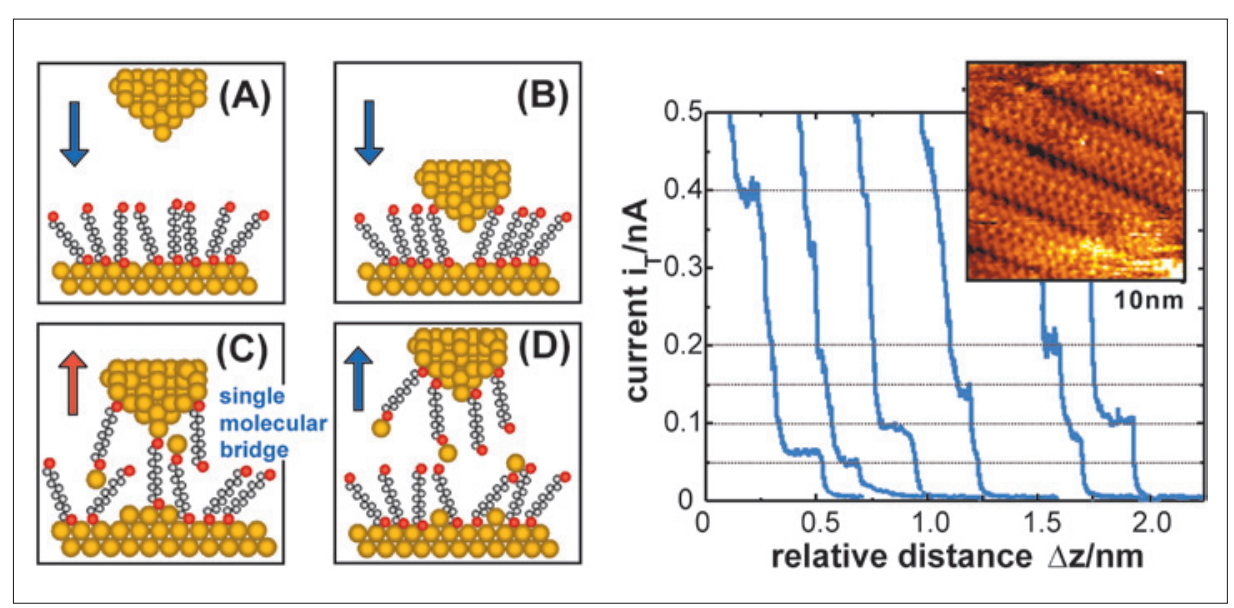

Fig. 2. Left panel: Schematic representation of the STM-type 'contact junction' approach. (A) Approach, (B) Formation of molecular contacts, (C) Pulling and (D) Breaking. Right panel: Typical current-distance retraction curves recorded with a gold STM tip for $0.1 \mathrm{mM} 1,9$-nonanedithiol in 1,3,5-trimethylbenzene on $\mathrm{Au}(111)-(1 \times 1)$, at $\mathrm{E}_{\text {bias }}=0.10 \mathrm{~V}$. The setpoint current before disabling the feedback was chosen at $\mathrm{i}_{\mathrm{T}}=100 \mathrm{pA}$. The pulling rate was $4 \mathrm{~nm} \mathrm{~s}{ }^{-1}$. The inset illustrates a high resolution STM image of the striped low-coverage nonanedithiole phase on $\mathrm{Au}(111)-(1 \times 1) ; \mathrm{i}_{\mathrm{T}}=0.1$ $\mathrm{nA}, \mathrm{E}_{\text {bias }}=0.1 \mathrm{~V}^{[35]}$
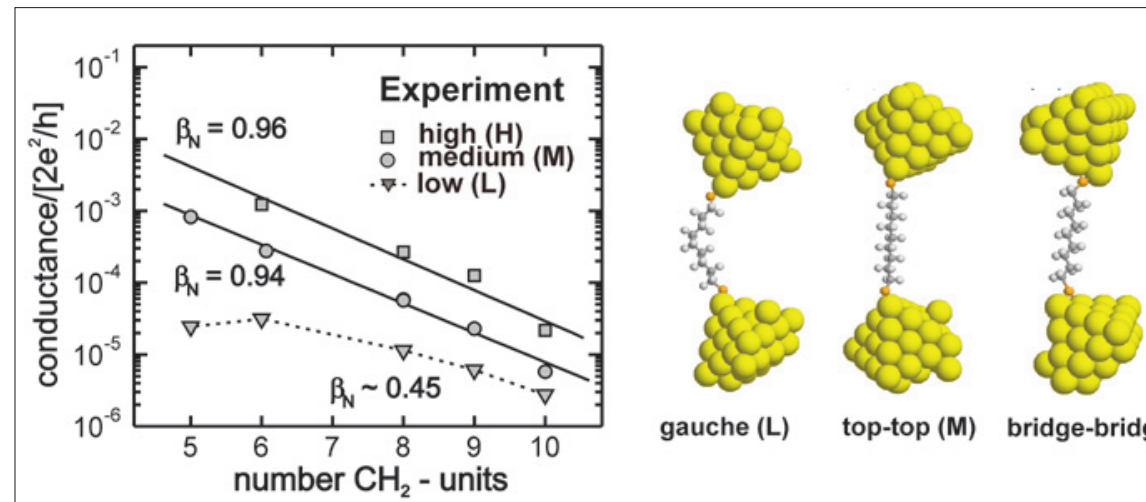

gauche (L)

top-top (M)

bridge-bridge $(\mathrm{L})$

Fig. 3. Left panel: Chain length dependence of the single junction Au | alkanedithiol | Au conductance in a semilogarithmic representation. The three sets of conductance values - high $(H)$, medium $(M)$ and low $(L)$ - are shown as squares, circles and triangles. The straight lines were obtained from a linear regression analysis with decay constants $\beta_{N}$ defined per methylene $\left(\mathrm{CH}_{2}\right)$ unit. Right panel: Three typical arrangements of a single alkanedithiol molecule bridged between Au electrodes: 1, 9-nonanedithiol (ND) with one gauche defect and both terminal sulfur atoms coordinated in atop position (low, L), ND in all-trans conformation and atop-atop coordination (medium, M), (C) all-trans ND in a bridge-bridge coordination (high, H). ${ }^{[35]}$

tact resistances, thin adjacent double layers $(<1 \mathrm{~nm})$ and high mobility of the charge carriers (ions). The magnitude of the field in the electrochemical double layer (EDL) is close to the gate field required to significantly modulate the current through a molecular-sized nanostructure according to first principle calculations by di Ventra et al. ${ }^{[64,65]}$

The electrochemical approach is unique as the measured current represents both the electrical contact to the external circuit and the functional state of the nanostructure (single molecule, nanocluster).

In the following sections we shall discuss selected examples of our own work on charge transport with tailored molecules and clusters. We present first a STM-based method to investigate charge transport characteristics in single metal | molecule | metal junctions at solid | liquid interfaces. The approach will be illustrated based on two case studies with $\alpha, \omega$-alkanedithiols ${ }^{[35]}$ and biphenyldithiols. ${ }^{[45,46]}$ Then we will discuss single redox-active nanojunctions in an electrochemical environment with viologen-type molecules bound either to one (asymmetric configuration) or to both (symmetrical configuration) adjacent electrodes. ${ }^{[20,63]}$ The molecular experiments will be complemented with an example on quantized double layer charging of gold nanoparticles. ${ }^{[66]}$

\section{Charge Transport in $\mathrm{Au}$ $\alpha, \omega$-alkanedithiol | Au Junctions at Solid | Liquid Interfaces}

Fig. 2 shows a schematic representation of the STM-based contact junctions approach as developed in our group and applied to explore charge transfer characteristics in gold $\mid \alpha, \omega$-alkanedithiol gold junctions.[35,67] The following strategy was applied: A sharp gold STM tip, capable of imaging experiments with atomic resolution, was brought to a preset tunneling position (A). Subsequently, the STM feedback was switched off, and the tip approached the adsorbate-modified substrate surface at constant $\mathrm{x}$ - $\mathrm{y}$ position. The approach was stopped typically before reaching point contact with the gold surface ('gentle touch', shown in panel (B)) or upon reaching point contact ('hard touch'). After a dwelling time of $100 \mathrm{~ms}$, sufficient to create molecular junctions between tip and substrate, the tip was retracted and the corresponding current-distance trace recorded. The entire cycle is typically repeated more than 1000 times.

The individual current-distance traces are non-exponential and show characteristic series of plateaus separated by abrupt steps (Fig. 2). The observed currents are 2 to 3 orders of magnitude smaller compared to metal nanocontacts. We ascribe these conductance steps to the breaking of individual respective multi-molecule junctions.

The statistical analysis of the plateau currents yields histograms with characteristic peaks. Careful analysis of these conductance peaks allows the identification of three distinctly different sequences of equally spaced maxima, which are attributed to low $\mathrm{L}$, medium $\mathrm{M}$ and high $\mathrm{H}$ conductance junctions. ${ }^{[35]}$ The logarithm of the single molecular junction conductance is linearly dependent on the chain length, with a decay constant $\beta \sim 1$ for the high and medium type junctions. Distinctly different results were obtained in the low conductance regime. A tentative value of $\beta$ 0.45 is estimated (Fig. 3).

Comparison with quantum chemistry $a b$ initio simulations demonstrated that the multiple conductance values of $\mathrm{Au}$ alkanedithiol | Au junctions could be attributed to different Au-sulfur coordination geometries and to different conformations of the alkyl chain. In particular, the 'medium' conductance values $M$ correspond to an all-trans conformation of the alkyl chain with one of the sulfur atoms coordinated in atop position to a single Au atom. The 'high' conductance values $H$ represent an all-trans alkyl chain in combination with both sulfurs coordinated to two Au atoms in bridge geometry. The sequence of 'low' conductance values 
$\mathrm{L}$ is attributed to isomers of alkanedithiols with gauche defects (Fig. 3).

\section{Chemically Controlled Conductance: Torsion-Angle Dependence in Single-Molecule Biphenyldithiol Junctions}

Employing the methodology developed in ref. [35], we investigated the conductance of a family of custom-made biphenyldithiol derivatives with conformationally fixed torsion angles to explore structure-property relationships at the single molecule level. ${ }^{45,46]}$ Biphenyl derivatives consisting of two aromatic rings interconnected by a single $\mathrm{C}-\mathrm{C}$ bond gained considerable interest as subunits for single molecular rectifying or switching systems as the two $\pi$-systems can be either in the same plane or perpendicular to each other representing 'on' or 'off' states, respectively. ${ }^{[37,67-70]}$

As displayed in Fig. 4, methyl side groups or an alkyl chain of various lengths connected at the 2,2'-positions of the biphenyl system adjust the resulting torsion angle $\phi$. In this series of compounds the length of the interring alkyl chain is the only structural variation, keeping the electronic structure of the biphenyl systems within this family of compounds as uniform as possible. The biphenyl conformation is locked by an intra-molecular bridge with the number of $-\mathrm{CH}_{2}$-units dictating the torsion angle $\phi$, lowering the expected motion and conformational variation of each molecule immobilized in the junction considerably. Due to their terminal sulfur anchor groups, the series of compounds forms a stable molecular junction.

The STM-based single molecule experiments revealed that the junction conductance depends on the torsion angle $\phi$ between the two phenyl rings: twisting the biphenyl system from flat $\left(\phi=0^{\circ}\right)$ to perpendicular $\left(\phi=90^{\circ}\right)$ decreased the conductance by a factor of 30 following a linear $\cos ^{2} \phi$ dependence. Detailed calculations based on density functional theory (DFT) revealed a HOMO-controlled transport mechanism and support the experimentally obtained $\cos ^{2} \phi$ correlation between the junction conductance $\mathrm{G}$ and the torsion angle $\phi$. A two level model as parameterized by the DFT calculations demonstrated that the transmission factorizes in an 'off-resonance' junction, such that 'ringto-ring' and 'electrode-ring' transmission components could be separated.[46] We found that for all molecules with $\phi \leq$ $80^{\circ}$ one $\pi$ channel dominates the transport. Contributions from degenerate $\sigma-\pi$ and $\pi-\sigma$ channels were only observed for biphenyl derivatives with completely broken conjugation, e.g. for $\phi=90^{\circ}$. Extending

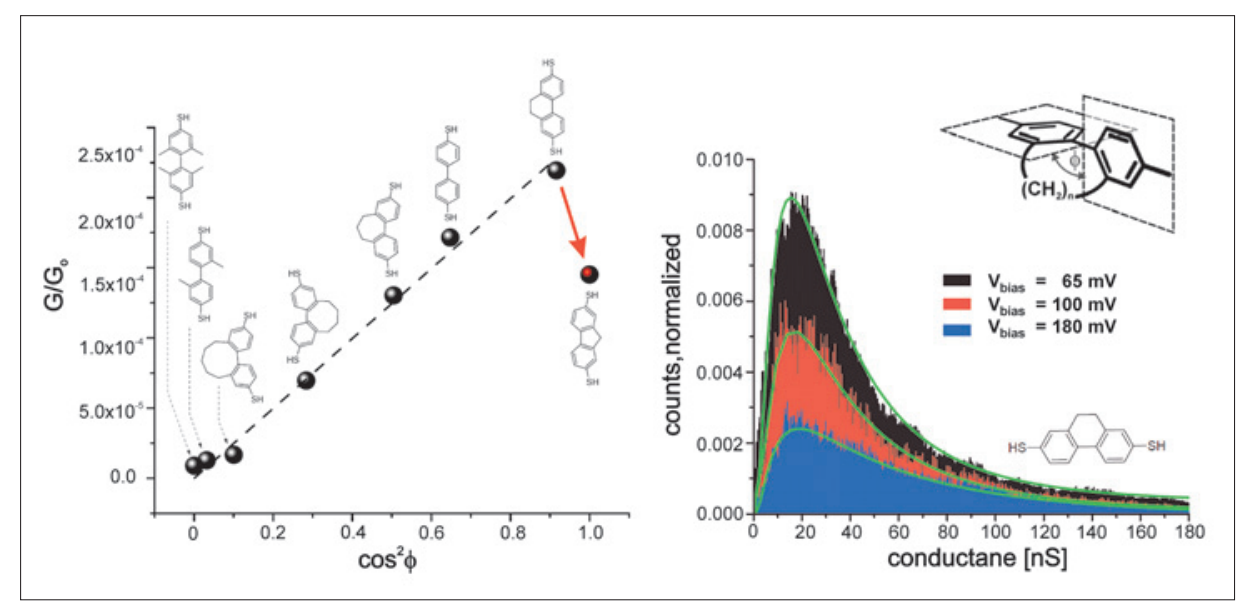

Fig. 4. Left panel: Experimentally determined single junction conductance of thiol-terminated biphenyl molecules vs $\cos ^{2} \phi$, where $\phi$ is the torsion angle of the molecules as given by the $X$-ray data. ${ }^{[45]}$ The dashed line represents the linear fit $G / G_{0}=a_{e x} \cdot \cos ^{2} \phi$ with $a_{e x}=2.4410^{-4}$ to all molecules studied, except the fluorene derivative. Right panel: Plateau-point histogram for the $\mathrm{C}_{2}$-bridged biphenyldithiol derivative, as recorded from individual current-distance traces in an STM-based break junction experiment (gold tip, $\mathrm{Au}(111)$ substrate; $\mathrm{E}_{\mathrm{bias}}=0.10 \mathrm{~V}$ ) at three different bias voltages in mesitylene. ${ }^{[46]}$

the experimental and theoretical studies to biphenyl families with amino-[37,70] and cyano-[71] anchoring groups revealed that the former exhibit HOMO-controlled transport characteristics while the latter is LUMO-controlled, respectively.

Our study provides a clear example of the reproducible measurement of a single molecule-specific property, the pronounced $\cos ^{2} \phi$ variation, its tuning by controlled synthetic chemistry, and the development of a molecular-level understanding by combination with a careful DFT-based analysis of transmission channels.

\section{Electrochemically Gate- controlled Electron Transport with Redox-active Molecules}

The development of the methodology to measure single molecule electron transport in metal | molecule | metal configurations motivated applications in an electrochemical environment employing the concept of 'electrolyte gating'.[52,53,63] These examples include porphyrines, ${ }^{[18]}$ viologens, ${ }^{[19,20,63,72,73]}$ aniline and thiophene oligomers, ${ }^{[74-77]}$ transition metal complexes, ${ }^{[78-80]}$ carotenes, ${ }^{[81]}$ nitro-derivatives of oligophenylene ethynelene, ${ }^{[82,83]}$ ferrocenes, $[66,84,85]$ perylene tetracarboxylic bisimides, ${ }^{[86-88]}$ and redox-active proteins. ${ }^{[89-92]}$ Despite these qualitative studies with various redox-active molecular hybride systems, there is still a considerable lack in understanding basic relationships between molecular structures and conductance properties.

In an attempt to contribute to a more advanced understanding of these phenomena, we have carried out recently several systematic experimental and theoretical studies with redox-active viologens, ${ }^{[20,63,72]}$ perylene bisimides ${ }^{[88]}$ and ferrocenes. ${ }^{[86]}$ As an example, we will describe in the following selected experiments employing gold nanojunctions modified with N,N'-bis(6-thiohexyl)-4,4'-bipyridinium bromide (HS-6V6-SH) or N-hexyl-N'(6-thiohexyl)-4,4'-bipyridinium bromide (HS-6V6), which are composed of the redox-active 4,4'-bipyridinium cation $\mathrm{V}^{2+}$ (viologen) as a core, two flexible alkyl spacer units attached to each ring nitrogen with either two or one terminal SH anchoring group. The latter ensures the chemical surface immobilization. ${ }^{20,63]}$

Depending on the applied potential, the redox-active viologen group exists in three different redox states, the oxidized and stable dication $\mathrm{V}^{2+}$, or an electron rich radical cation $\mathrm{V}^{+}$respective the neutral species $\mathrm{V}^{\circ}$. We shall focus on the potentialinduced reversible transition between the dication $\mathrm{V}^{2+}$ and the radical cation $\mathrm{V}^{+}$.

Fig. 5 shows a typical current-potential curve (cyclic voltammogram) for HS-6V6$\mathrm{SH}$ on $\mathrm{Au}(111)$ for a low and a densely packed high coverage molecular adlayer. The latter is disordered. At low coverages, however, a highly ordered zig-zag layer of flat lying viologen-type molecules could be identified with submolecular resolution of the sulfur atoms, the alkyl chains and the viologen moiety. The low coverage phase was chosen to explore the single molecular conductance as a function of the redox state.

The STM break junction approach, as introduced before, ${ }^{[35,67]}$ was now applied under strict electrochemical potential control.[20] The recorded retracting currentdistance traces revealed characteristic pla- 


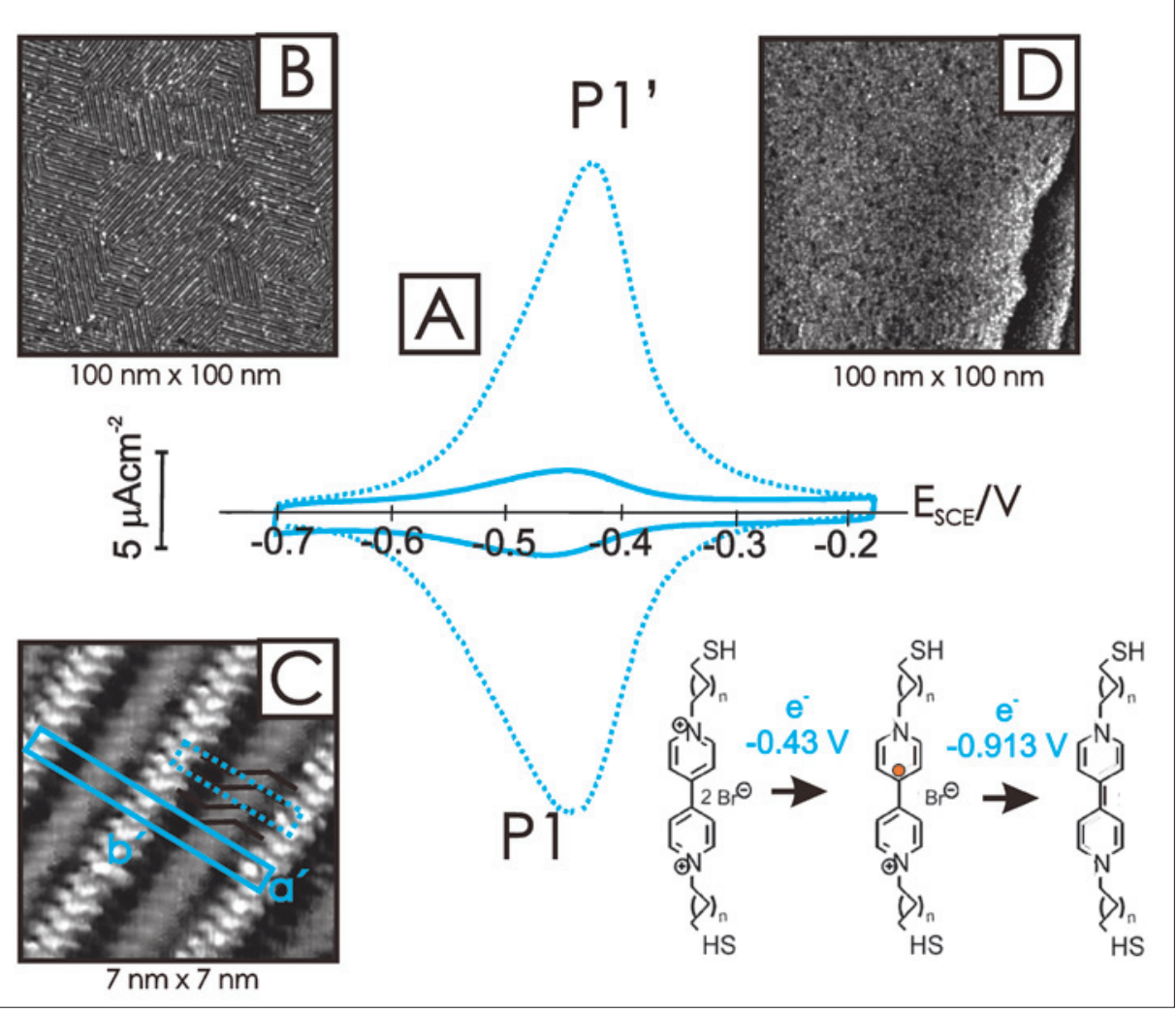

Fig. 5. (A) Cyclic voltammogram of an $\mathrm{Au}(111)-(1 \mathrm{x} 1)$ electrode in $0.05 \mathrm{M} \mathrm{KClO}_{4}, \mathrm{pH} \sim 7$, modified with a low coverage (solid line) and a high coverage (dotted line) adlayer of HS-6V6-SH for the reversible one-electron oxidation/reduction between the viologen dication $\mathrm{V}^{2+}$ and the radical cation $\mathrm{V}^{+}$form. (B) Large scale in situ STM image of the low coverage striped phase of HS-6V6$\mathrm{SH}, \mathrm{E}_{\mathrm{S}}=-0.25 \mathrm{~V}, \mathrm{E}_{\text {bias }}=-0.10 \mathrm{~V}, \mathrm{i}_{\mathrm{T}}=60 \mathrm{pA}$; (C) high resolution image of the striped phase, $\mathrm{E}_{\mathrm{S}}=$ $-0.35 \mathrm{~V}, \mathrm{E}_{\text {bias }}=0.08 \mathrm{~V}, \mathrm{i}_{\mathrm{T}}=40 \mathrm{pA}$; (D) high coverage adlayer of HS-6V6-SH, $\mathrm{E}_{\mathrm{S}}=-0.25 \mathrm{~V}, \mathrm{E}_{\text {bias }}=$ $-0.09 \mathrm{~V}, \mathrm{i}_{\mathrm{T}}=60 \mathrm{pA} .{ }^{[20]}$

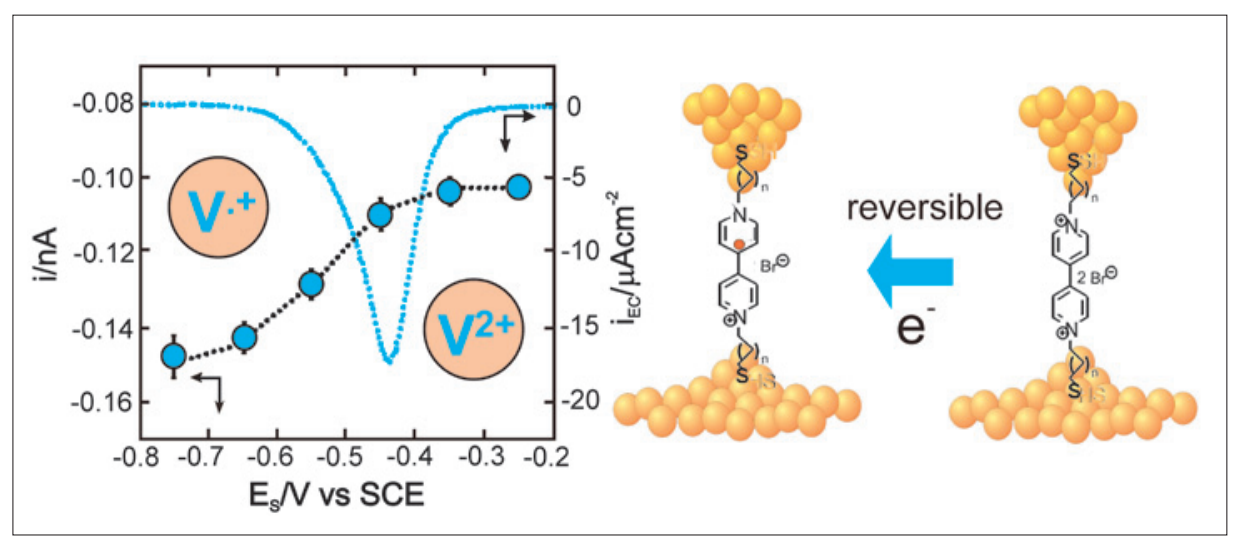

Fig. 6. Plot of the single molecule conductance currents i of $\mathrm{Au}(\mathrm{T})|\mathrm{HS}-6 \mathrm{~V} 6-\mathrm{SH}| \mathrm{Au}(\mathrm{S})$ junctions vs. the substrate potential $E_{S}$ at fixed bias $\left(E_{S}-E_{T}\right)=-0.100 \mathrm{~V}$. Each data point was obtained from conductance histograms as constructed from several hundreds of individual current-distance pulling curves. The dotted line corresponds to the macroscopic current-voltage curve for the reduction of $\mathrm{V}^{2+} \rightarrow \mathrm{V}^{+\bullet}$ (c.f. cyclic voltammograms in Fig. 5). ${ }^{[20]}$

teaus of 0.2 to $0.3 \mathrm{~nm}$ separated by abrupt steps. The statistical analysis of these stretching curves leads to single molecular conductance data of the oxidized and reduced form of the viologen bridge by appropriate substrate and tip polarization (Fig. 6). The single molecular conductance of the HS-6V6-SH junctions is rather constant in the stability range of the oxidized viologen dication $\mathrm{V}^{2+}$, and increases at more negative potentials by $c a .50 \%$ until a plateau is reached at $\mathrm{E} \leq-0.700 \mathrm{~V}$, e.g. past the formal potential of the one-electron redox process. This trend in single-molecule redox switching is attributed to the higher electron density and the higher degree of conjugation of the radical cation as compared to the dication. ${ }^{20]}$ Quantum chemical calculations of Bagrets et al. suggested additional contributions from interactions of the tunneling electrons with molecular vibrations. ${ }^{[91]}$

We note that a particular large effect upon electrolyte gating was observed for molecular junctions formed with thioland pyridyl-group terminated perylene bisimide derivatives attached to two gold electrodes. ${ }^{[88]}$ Here we found an increase in the single molecule conductance of up to 100 , i.e. from $1 \mathrm{nS}$ to $~ 100 \mathrm{nS}$, upon reduction of the redox-active perylene unit in aqueous $\mathrm{LiClO}_{4}$ as electrolyte.

Besides the above transport experiment in a symmetric Au HS-6V6-SH $\mathrm{Au}$ junction, we also developed a single molecule tunneling experiment. ${ }^{[20,63,72]}$ Here, a redox-molecule modified tunneling junction is created with an asymmetric viologen HS-6V6 bound either to the $\mathrm{Au}$ tip or to the $\mathrm{Au}(111)$ substrate surface (Fig. 7). After establishing stable tunneling junctions, the STM feedback was switched off, and subsequently we recorded at constant xyz position the tunneling current as a function of the applied voltage while keeping the voltage difference between tip and substrate (bias voltage) constant. In other words, the Fermi levels of tip and substrate were shifted relative to the discrete molecular levels. In both cases, i.e. a viologen-modified tip or substrate, an enhanced tunneling current with a well-defined maximum close to the equilibrium potential $\mathrm{E}^{\circ}$ of the redox couple was observed, which is attributed to the 'opening' or 'gating' of an additional molecular tunneling channel and resembles a transistor-like behavior. The experimental observations could be quantitatively explained by a sequential two-step electron transfer process accompanied with partial vibration relaxation. ${ }^{[63]}$ This model was recently developed by Kuznetsov and U1strup. ${ }^{[52,53]}$ Our analysis leads to characteristic parameters, such as reorganization energy, potential drop, and overpotential across the tunneling gap, and demonstrates that the magnitude of the tunneling enhancement depends on the initial redox state of HS6V6 $\left(\mathrm{V}^{2+}\right.$ or $\left.\mathrm{V}^{+\bullet}\right)$.

Complementary tunneling spectroscopic experiments were also carried out in variable bias mode with a fixed substrate potential and the tip potential varied in a wide range. The resulting current response is asymmetric and resembles the characteristics of a diode. ${ }^{[63]}$ At high tip potentials a small current due to direct tunneling at fixed gap geometry is observed, while an enhanced exponential-like tunneling response is found at negative tip potentials due to the opening of a redox-mediated tunneling channel. As an example, the 'on/ off ratio' between the negative and the corresponding positive branches of the tunneling current-voltage curve at a bias voltage of $\pm 0.4 \mathrm{~V}$ is $c a .20$. 

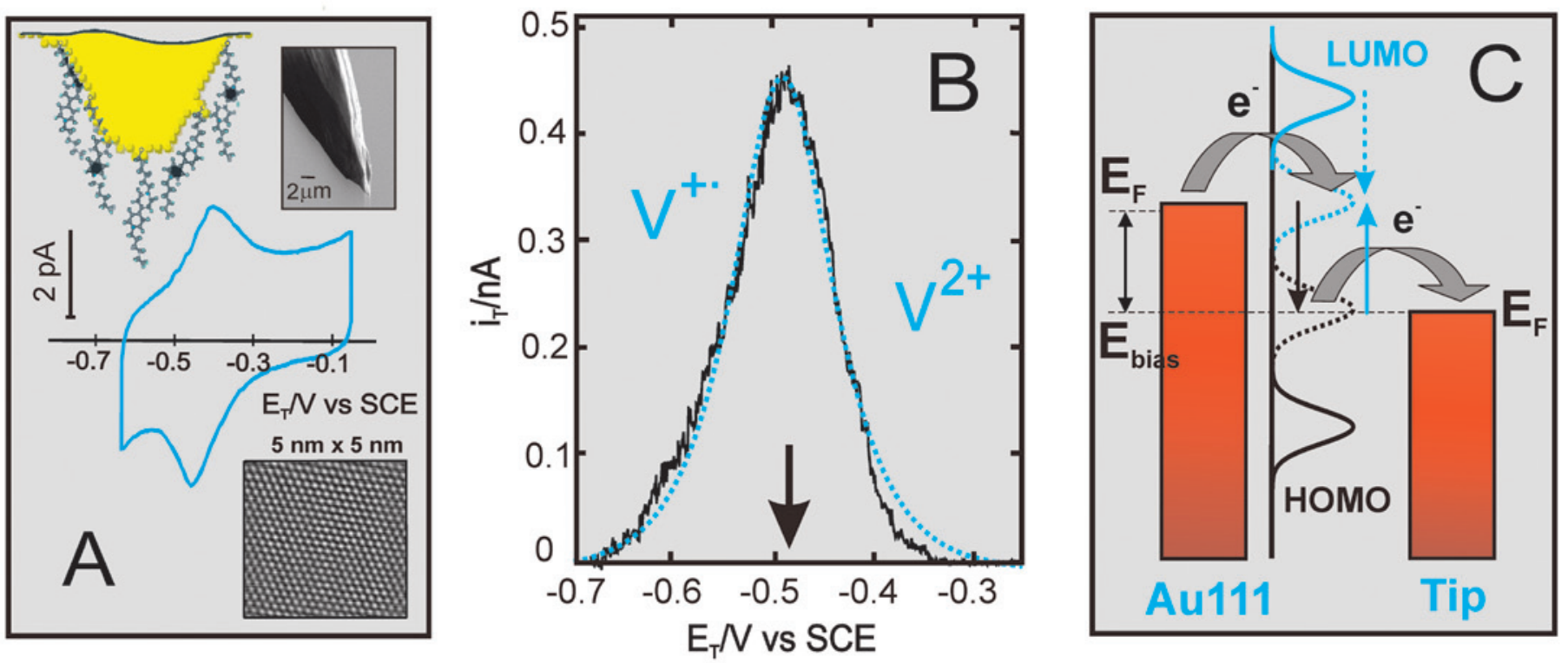

Fig. 7. (A) Set-up of the redox-mediated tunneling experiment with a viologen-modified Au tip; (B) Average $\mathrm{i}_{\mathrm{T}}$ vs. $\mathrm{E}_{\mathrm{S}}$ curves recorded in constant bias spectroscopy mode, $\mathrm{i}_{\mathrm{T}}=0.1 \mathrm{nA}, \mathrm{E}_{\text {bias }}=0.050 \mathrm{~V}$, after base line correction. The sweep started in the stability region of $\mathrm{V}^{+}$. The dotted blue line represents the fit using the Kuznetsov/Ulstrup model of sequential two-step electron transfer with partial vibration relaxation. ${ }^{20,52,53,63]}$. (C) Schematic energy level diagram of a two-step electron transfer process mediated by a redox-active molecule. The electron is transferred from the Fermi level of the substrate (left) $\mathrm{E}_{\mathrm{S}}$ to the LUMO of the molecule and after partial vibrational relaxation to the Fermi level of the tip $\mathrm{E}_{\mathrm{T}}$ (right).

We would like to point out that the asymmetric Au | HS-6V6 | Au configuration is distinctly different from the symmetric $\mathrm{Au} \mid$ molecule |Au configuration. ${ }^{63]}$ The latter exhibits modifications of the junction conductance due to structure changes of the redox-active unit ('inner sphere reorganization energy'), while the former exhibits a maximum of the tunneling current due to the coexistence of the oxidized and the reduced forms with dominant contributions of the dynamics of the solvent molecules in the gap ('outer sphere reorganization energy'). We also like to note that the observed enhancement of the tunneling current in an electrochemical environment is not restricted to viologenbased junctions.

\section{From Redox Gating to Quantized Charging of Nanometer-sized Gold Clusters}

Metal nanoparticles (NP) represent another typical nanoscale object with unique electronic, optical, catalytic and magnetic properties, which are distinctly different from both molecular and bulk regimes. ${ }^{\text {[93] }}$ Their electronic and electrochemical properties can be tuned through metal core and ligand shell. For instance, Au-NPs with a core diameter $\leq 1 \mathrm{~nm}$ behave like a similar sized molecule with a discrete electronic spectrum at a wide HOMO-LUMO gap. Bulk properties are observed for larger NP

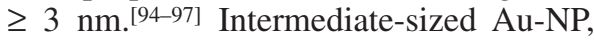
1-3 nm, display electrochemically detect- able quantum confinement effects such as quantized double layer charging. ${ }^{[96-105]}$ This observation implies that, within the stability range of the clusters, multiple accessible and addressable electronic levels exist.

Chen et al. reported on quantized double layer charging based on macroscopic voltammetric experiments with a monolayer of alkanethiol-protected Au-NP immobilized on a Au(poly) substrate. [106] Single electron Coulomb charging was observed for different types and sizes of individual metal nanoparticles in vacuum or in air employing specifically STM and STS. ${ }^{[11,99,107-109]}$ Albrecht et al. showed in a proof-of-principle experiment the possibility of intrinsic multi-state switching of $\mathrm{Au}$ nanoclusters through electrochemical gating. [110]

Motivated by this pioneering report we created a thiol-based self-assembled template monolayer on $\mathrm{Au}(111)$ electrodes with immobilized and uniformly distributed gold cluster of $2.4 \pm 0.5 \mathrm{~nm}$ diameter and a rather narrow size distribution (Fig. 8). ${ }^{[66]}$ Constant bias mode electrochemical scanning tunneling spectroscopy (STS) experiments with the gold tip positioned above a single gold cluster (asymmetric tunneling junction) revealed up to seven clearly resolved, rather narrow current peaks with an equal peak-to-peak spacing of $0.11 \pm$ $0.02 \mathrm{~V}$. Based on the distribution of peak spacings and peak heights we attribute our observations to a modulation of the tunneling current by sequential capacitive charging of single gold nanoclusters. Following this hypothesis we estimate ${ }^{[96,97,100,101]}$ the average particle capacitance $\mathrm{C}=\mathrm{e} / \Delta \mathrm{E}=$ $1.5 \pm 0.2 \mathrm{aF}$. The observed feature of sequential capacitive charging is reminiscent of a successive electronic charging of redox molecules through several oxidation/ reduction states. However, the latter are usually much wider than the former.

\section{Summary and Outlook}

We have presented and discussed in this paper selected examples of our own work on charge transport in gold | (single) molecule | gold junctions at solid | liquid interfaces employing a scanning tunneling microscopy (STM)-based 'break junction' technique. After an introduction of the experimental technique we discussed four case studies with 'inactive' $\alpha, \omega$-alkanedithiols and biphenyldithiols, 'redox-active' thio-alkylated viologens and surface immobilized gold nanoclusters in the quantum confinement range. The combination of these reproducible and statistical relevant single-molecule measurements with quantum chemistry and transport calculation revealed important new aspects in the understanding of basic relationships between molecular structure and conductance properties. In particular, the observations on the intrinsic electronic properties of locally addressable single 'redox-molecules' and gold nanoclusters employing the concept of 'electrolyte gating' open new experimental and theoretical perspectives toward multi-state electronic switch- 


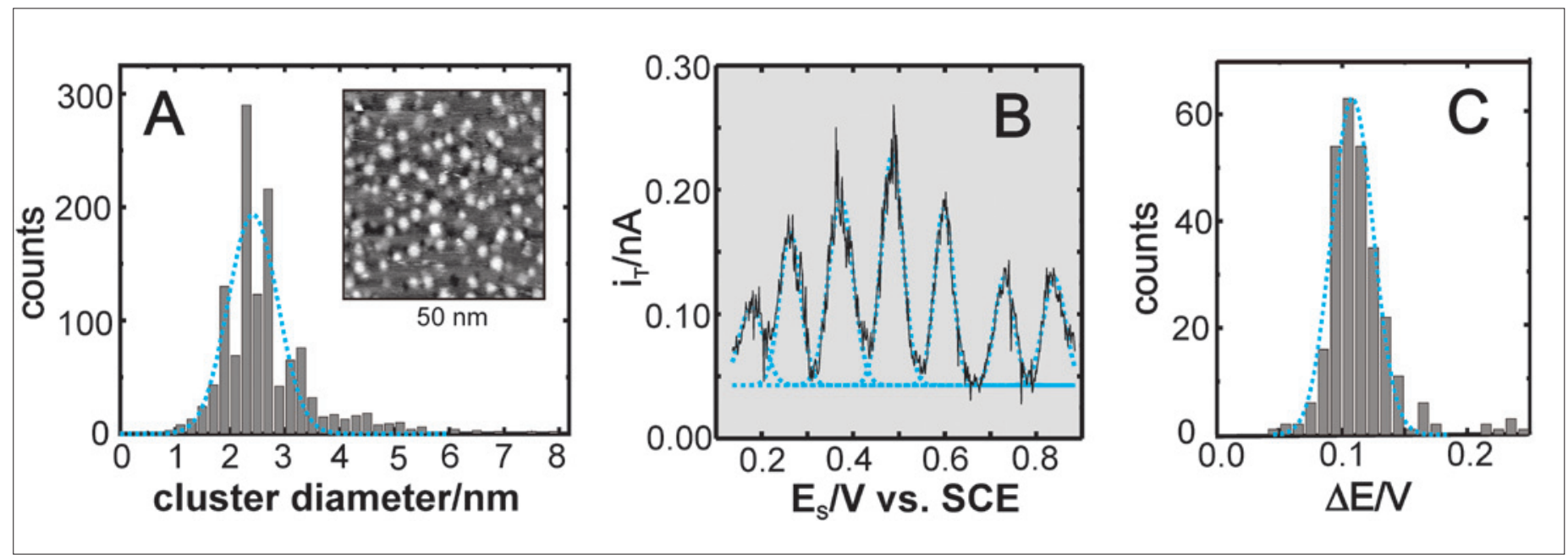

Fig. 8. (A) STM image $\left(i_{T}=0.05 n A, E_{S}=0.7 \mathrm{~V}, E_{T}=0.1 \mathrm{~V}\right.$; vertical scale $\left.0.4 \mathrm{~nm}\right)$ and size distribution histogram of gold nano-clusters formed within a self-assembled template monolayer on $\mathrm{Au}(111)$ in $1.0 \mathrm{M} \mathrm{HClO}_{4}$. The dotted line represents the Gaussian fit. The corresponding parameters are the average cluster diameter $2.42 \mathrm{~nm}$ and the standard deviation $\sigma=0.45 \mathrm{~nm}$. (B) Example of a constant bias current-voltage response of a single gold cluster as triggered by 'electrolyte gating', $\mathrm{E}_{\text {bis }}=0.1 \mathrm{~V}, \mathrm{i}_{\mathrm{T}}=0.05 \mathrm{nA}$. The dotted traces represent its fit by a series of Gaussians. (C) Peak spacing histogram and Gaussian fit for a data set 361 individual traces, such as shown in (B). The corresponding parameters are the average spacing $\Delta \mathrm{E}=$ $0.11 \mathrm{~V}$ and $\sigma=0.017 \mathrm{~V}$

ing, current amplification, rectification or other nanoscale electronic functions in condensed media at room temperature. These studies may also open up unique opportunities in the newly developing field of nanoscale molecular electrochemistry.

\section{Acknowledgement}

This work was supported by the Swiss National Science Foundation, the Volkswagen Foundation, FUNMOLS and the DFG priority program 1243 . The authors particularly like to acknowledge the fruitful discussions with $\mathrm{M}$. Mayor, and with the theory groups of F. Evers and F. Pauly.

Received: May 3, 2010

[1] A. Aviram, M. Ratner, Chem. Phys. Lett. 1974, 29, 277.

[2] C. Joachim, M. A. Ratner, Proc. Natl. Acad. Sci. USA 2005, 102, 8801.

[3] A. Nitzan, M. A. Ratner, Science 2003, 300, 1384

[4] J. R. Heath, Annu. Rev. Mater. Res. 2009, 39, 1.

[5] J. C. Cuevas, E. Sheer, 'Molecular Electronics: An Introduction to Theory and Experiment', World Scientific, 2010

[6] R. L. Carroll, C. Gorman, Angew. Chem. Int Ed. 2002, 41, 4378.

[7] R. M. Metzger, J. Mater. Chem. 2008, 18, 4364

[8] S. J. van der Molen, P. Liljeroth, J. Phys. Cond. Mater. 2010, 22, 133001

[9] F. Chen, J. Hihath, Z. Huang, X. Li, N. Tao, Annu. Rev Phys. Chem 2007, 58, 535 .

[10] Z. J. Donnhauser, B. A. Mantooth, K. F. Kelly, L. A. Bumm, J. D. Monnell, J. J. Stapleton, D W. Price, A. M. Rawlett, D. L. Allara, J. M. Tour, P. S. Weiss, Science 2001, 292, 2303.

[11] R. P. Andres, T. Bein, M. Dorogi, S. Teng, J. I. Hendersen, C. P. Kubiak, W. Mahoney, R. G. Osifchin, R. Reifenberger, Science 1996, 272, 1323.

[12] J. K. Gimzewski, C. Joachim, Science 1999, 283,1683

[13] J. Repp, G. Meyer, S. Paavilainen, F. E. Olsson, M. Persson, Science 2006, 312, 1196.
[14] D. J. Wold, R. Haag, M. A. Rampi, C. D. Frisbie, J. Phys. Chem. B 2002, 106, 2813.

[15] X. D. Cui, A. Primak, X. Zarate, J. Tomfohr, O. F. Sankey, A. L. Moore, T. A. Moore, D. Gust, G. Harris, S. M. Lindsay, Science 2001, 294 , 571.

[16] F. R. F. Fan, J. Yang, L. Cai, D. W. Price, S. M. Dirk, D. V. Kosynkin, Y. Yao, A. M. Rawlett, J. M. Tour, A. J. Bard, J. Am. Chem. Soc. 2002, $124,5550$.

[17] B. Xu, N. J. Tao, Science 2003, 301, 1221

[18] N. J. Tao, Phys. Rev. Lett. 1996, 76, 4066.

[19] W. Haiss, H. van Zalinge, S. J. Higgins, D. Bethell, H. Höbenreich, D. J. Schiffrin, R. J. Nichols, J. Am. Chem. Soc. 2003, 125, 15294.

[20] Z. Li, B. Han, G. Meszaros, I. Pobelov, T. Wandlowski, A. Blaszczyk, M. Mayor, Faraday Discuss. 2006, 131, 121.

[21] D. S. Seferos, S. A. Trammell, G. C. Bazan, J. G. Kushmerick, Proc. Natl. Acad. Sci. USA $\mathbf{2 0 0 5}, 102,8821$

[22] T. Dadosh, Y. Gordin, R. Krahne, I. Khivrich, D. Mahalu, Nature 2005, 436, 677.

[23] J. Liao, L. Bernard, M. Langer, C. Schönenberger, M. Calame, Adv. Mat. 2006, 18 , 2444.

[24] M. A. Reed, C. Zhou, C. J. Muller, T. P. Burgin, J. M. Tour, Science 1997, 278, 252.

[25] C. Kergueris, J. P. Bourgoin, S. Palacin, D. Esteve, C. Urbina, M. Magoga, C. Joachim, Phys. Rev. B 1999, 59, 12505.

[26] J. Reichert, R. Ochs, D. Beckmann, H. B. Weber, M. Mayor, H. v. Löhneysen, Phys. Rev. Lett. 2002, 88, 176804 .

[27] M. T. Gonzalez, S. Wu, R. Huber, S. J. van der Molen, C. Schönenberger, M. Calame, Nano Lett. 2006, 6, 2238.

[28] E. Lörtscher, J. W. Ciszek, J. Tour, H. Riel, Small 2006, 2, 973.

[29] J. Park, A. N. Pasupathy, J. I. Goldsmith, C. Chang, Y. Yaish, J. R. Petta, M. Rinkoski, J. P. Sethna, H. D. Abruna, P. L. McEuen, D. C. Ralph, Nature 2002, 417, 722.

[30] M. A. Osorio, T. Bjornholm, J. M. Lehn, H. S. J. van der Zant, J. Phys. Cond. Mat. 2008, 20 , 374121

[31] J. Chen, M. A. Reed, A. M. Rawlett, J. Tour, Science 1999, 286, 1550.

[32] E. Tran, M. Duati, G. M. Whitesides, M. A. Rampi, Faraday Discuss. 2006, 131, 197.
[33] A. Salomon, D. Cahen, S. Lindsay, J. Tomfohr, V. B. Engelkes, C. D. Frisbie, Adv. Mat. 2003 $15,1881$.

[34] S. M. Lindsay, M. A. Ratner, Adv. Mat. 2007, $19,23$.

[35] C. Li, I. Pobelov, T. Wandlowski, A. Bagrets, A Arnold, F. Evers, J. Am. Chem. Soc. 2008, 130 , 318.

[36] R. Huber, M. T. Gonzalez, S. Wu, M. Langer, S. Grunder, V. Horboiu, M. Mayor, M. R. Bryce, C. Wang, R. Jitchati, C. Schönenberger, M. Calame, J. Am. Chem. Soc. 2008, 130, 1080.

[37] M. S. Hybertsen, L. Venkataraman, J. E. Klare, A. C. Whallery, M. L. Steigerwald, C. Nuckols, J. Phys. Cond. Mat. 2008, 20, 374115.

[38] F. Chen, X. Li, J. Hihath, Z. Huang, N. J. Tao, J. Am. Chem. Soc. 2006, 128, 15874

[39] S. Martin, W. Haiss, S. Higgins, P. Cea, M. C Lopez, R. J. Nichols, J. Phys. Chem. C 2008, $112,3941$.

[40] M. Kiguchi, S. Miura, K. Hara, M. Sawamura, K. Murakoshi, Appl. Phys. Lett. 2006, 89, 213104

[41] B. Kim, J. M. Beebe, Y. Jun, X. Y. Zhu, C. D. Frisbie, J. Am. Chem. Soc. 2006, 128, 7746

[42] S. Yasuda, S. Yoshida, J. Sasaki, Y. Okutsu, T. Nakamura, A. Taninka, O. Takeuchi, H. Shikegawa, J. Am. Chem. Soc. 2006, 128 7746.

[43] E. Leary, S. J. Higgins, H. van Zalinge, W. Haiss, R. J. Nichols, Chem. Commun. 2007, 3939.

[44] W. Haiss, C. Wang, R. Jitchati, I. Grace, S Martin, A. S. Batsanov, S. J. Higgins, M. R. Bryce, C. J. Lambert, P. S. Jensen, R. J. Nichols, J. Phys. Cond. Mater. 2008, 20, 374119.

[45] D. Vonlanthen, A. Mishchenko, M. Elbing, M Neuburger, T. Wandlowski, M. Mayor, Angew Chem. Int. Ed. 2009, 48, 8886.

[46] A. Mishchenko, D. Vonlanthen, V. Meded, M. Bürkle, C. Li, I. V. Pobelov, A. Bagrets, J. K. Viljas, F. Pauly, F. Evers, M. Mayor, T. Wandlowski, Nano Lett. 2010, 10, 156.

[47] G. J. Ashwell, M. Sujkoa, A. Green, Farad. Discuss. 2006, 131, 23

[48] M. Elbing, R. Ochs, M. Koentopp, M. Fischer, C. v. Hänisch, F. Weigand, F. Evers, H. B. Weber, M. Mayor, Proc. Natl. Acad. Sci. USA $\mathbf{2 0 0 5}, 102,8815$

[49] C. P. Collier, E. Wong, M. Belohradsy, F. M. Raymo, J. F. Stoddart, P. J. Kuekes, R. S. 
Williams, J. R. Heath, Science 1999, 285, 391.

[50] Y. Luo, C. P. Collier, J. O. Jeppesen, K. A. Nielsen, E. Delonno, G. Ho, J. Perkins, H. R. Tseng, T. Yamamoto, J. F. Stoddart, J. R. Heath, ChemPhysChem 2002, 3, 519.

[51] X. Duan, Y. Huang, C. M. Lieber, Nano Lett. 2002, 2, 487 .

[52] J. Zhang, Q. Chi, A. M. Kuznetsov, A. G. Hansen, H. Wackerbarth, H. Christensen, J. E. T. Andersen, J. Ulstrup, J. Phys. Chem. B $\mathbf{2 0 0 2}$ 106, 1131.

[53] J. Zhang, A. M. Kuznetsov, I. G. Medvedev, Q. Chi, T. Albrecht, P. S. Jensen, J. Ulstrup, Chem. Rev. 2008, 108, 2737.

[54] H. S. White, G. P. Kittlesen, M. S. Wrighton, J. Am. Chem. Soc. 1984, 106, 5375.

[55] E. A. Meulenkamp, J. Phys. Chem. B 1999, 103, 7831.

[56] M. Krüger, M. R. Buitelaar, T. Nussbaumer, C. Schönenberger, L. Forro, Appl. Phys. Lett. 2001, 78, 1291.

[57] S. Rosenblatt, Y. Yaishi, J. Park, J. Gore, V. Sazonova, P. L. McEuen, Nano Lett. 2002, 2, 869.

[58] H. X. He, J. S. Zhu, N. J. Tao, L. A. Nagahara, I. Amlani, R. Tsui, J. Am. Chem. Soc. 2001, 123, 7730; Phys. Rev. B. 2003, 68, 045302.

[59] B. Xu, X. Xiao, X. Zang, L. Yang, N. J. Tao, J. Am. Chem. Soc. 2005, 127, 2386.

[60] A. M. Kuznetsov, I. G. Medvedev, J. Ulstrup, J. Chem. Phys. 2007, 127, 104708.

[61] F. Chen, J. He, C. Nuckolis, T. Roberts, J. F. Klare, S. Lindsay, Nano Lett. 2005, 5, 563.

[62] I. N. Huela, H. B. Brom, A. J. Houtepen, D. Vanmaekelbergh, J. J. Kelley, A. A. Meulenkamp, Phys. Rev. Lett. 2004, 93, 166601.

[63] I. V. Pobelov, Z. Li, T. Wandlowski, J. Am. Chem. Soc. 2008, 130, 16045.

[64] M. di Ventra, S. T. Pantelides, N. D. Lang, Appl. Phys. Lett. 2000, 76, 3448.

[65] M. di Ventra, N. D. Lang, S. T. Pantelides, Chem. Phys. 2002, 281, 189.

[66] Z. Li, Y. Liu, S. F. L. Mertens, I. V. Pobelov, T. Wandlowski, J. Am. Chem. Soc., DOI: 10.1021/ ja102754n.

[67] G. Meszaros, C. Li, I. Pobelov, T. Wandlowski, Nanotechnology 2007, 10, 424004.

[68] L. Venkataraman, J. E. Klare, C. Nuckolls, M. S. Hybertsen, M. L. Steigerwald, Nature 2006, 442, 904.

[69] W. Haiss, C. Wang, R. Jitchati, I. Grace, M. Santiago, A. S. Batsanov, S. J. Higgins, M. R. Bryce, C. J. Lambert, P. S. Jensen, R. J. Nichols, J. Phys.: Condens. Matter. 2008, 20, 374119.

[70] F. Pauly, J. K. Viljas, J. C. Cuevas, G. Schön, Phys. Rev. B 2008, 77, 155312.

[71] H. Kondo, J. Nara, H. Kino, T. Ohno, J. Phys. Chem. 2008, 128, 064701.
[72] Z. Li, I. Pobelov, B. Han, T. Wandlowski, A. Blaszczyk, M. Mayor, Nanotechnology 2007, 18, 044018 .

[73] W. Haiss, T. Albrecht, H. van Zalinge, S. J. Higgins, D. Bethell, H. Höbenreich, D. J. Schiffrin, R. J. Nichols, A. M. Kuznetsov, J. Zhang, Q. Chi, J. Ulstrup, J. Phys. Chem. B 2007, 111, 6703 .

[74] H. He, J. Zhu, N. J. Tao, L. A. Nagahara, I. Amlani, R. Tsui, J. Am. Chem. Soc. 2001, 123, 7730.

[75] F. Chen, J. He, C. Nuckols, T. Roberts, J. E. Klare, S. Lindsay, Nano Lett. 2005, 5, 503

[76] B. Q. Xu, X. L. Li, X. Y. Xiao, H. Sakaguchi, N. J. Tao, J. Am. Chem. Soc. 2005, 5, 1491.

[77] J. He, F. Chen. S. M. Lindsay, C. Nuckols, Appl. Phys. Lett. 2007, 90, 072112.

[78] T. Albrecht, A. Guckian, J. Ulstrup, J. Vos, Nano Lett. 2005, 5, 1451.

[79] T. Albrecht, A. Guckian, A. M. Kuznetsov, J. Vos, J. Ulstrup, J. Am. Chem. Soc. 2006, 128, 17132.

[80] T. Albrecht, K. Moth-Poulsen, J. B. Christensen, J. Hjelm, T. Bjornholm, J. Ulstrup, J. Am. Chem. Soc. 2006, 128, 6574.

[81] I. Visoly-Fisher, K. Daie, Y. Terazono, C Herrero, F. Fungo, L. Otero, E. Durantini, J. J. Silber, L. Sereno, D. Gust, T. A. Moore, A. L. Moore, S. M. Lindsay, Proc. Natl. Acad. Sci. USA 2006, 103, 8686.

[82] X. Xiao, L. A. Nagahara, A. M. Rawlett, N. J. Tao, J. Am. Chem. Soc. 2005, 127, 9235.

[83] J. He, Q. Fu, S. M. Lindsay, J. W. Ciszek, J. M. Tour, J. Am. Chem. Soc. 2006, 128, 14828.

[84] R. A. Wassel, G. M. Credo, R. R. Fuierer, D. L. Feldheim, C. B. Gorman, J. Am. Chem. Soc. 2004, 126, 295.

[85] X. Xiao, D. Brune, J. He, S. Lindsay, C. B. Gorman, N. J. Tao, Chem Phys 2006, 326, 138.

[86] B. Xu, X. Xiao, X. Yang, L. Zang, N. J. Tao, J. Am. Chem. Soc. 2005, 127, 2386.

[87] X, Li, J. Hihath, F. Chen, T. Masuda, L. Zang, N. J. Tao, J. Am. Chem. Soc. 2007, 129 , 11535.

[88] C. Li, A. Mishchenko, Z. Li, I. Pobelov, T. Wandlowski, X. Q. Li, F. Würthner, A. Bagrets, F. Evers, J. Phys. Condens. Matter 2008, 20, 374122 .

[89] A. Alessandrini, M. Salerno, S. Frabboni, P. Facci, Appl. Phys. Lett. 2005, 86, 133902.

[90] Q. Chi, O. Farver, J. Ulstrup, J., Proc. Natl. Acad. Sci. USA 2005, 102, 16203.

[91] A. Alessandrini, S. Corni, P. Facci, Phys. Chem. Chem. Phys. 2006, 8, 4383.

[92] Q. Chi, J. Zhang, P. S. Jensen, H. E. M. Christensen, J. Ulstrup, Faraday Discuss. 2006, $131,181$.

[93] G. Schmid, 'Nanoparticles', Wiley-VCH, Weinheim, 2004.
[94] M. C. Daniel, D. Astruc, Chem. Rev. 2004, 104, 293.

[95] A. Z. Kosousi, A. A. Dhirani, Chem. Rev. 2008, 108, 4072.

[96] R. W. Murray, Chem. Rev. 2008, 108, 2688.

[97] A. C. Templeton, W. P. Wuelfing, R. W. Murray, Acc. Chem. Res. 2000, 33, 27

[98] S. Chen, R. S. Ingram, M. J. Hosteler, J. J. Pietron, R. W. Murray, T. G. Schaaff, J. T. Khoury, M. M. Alvarez, R. L. Whetten, Science 1998, 280, 2098.

[99] S. Ingram, M. J. Hosteler, R. W. Murray, T. G. Schaaff, J. T. Khoury, R. L. Whetten, T. P. Bigioni, D. K. Guthrie, P. N. First, J. Am. Chem. Soc. 1997, 119, 9279.

[100] S. Chen, R. W. Murray, S. W. Feldberg, J. Phys. Chem. B 1998, 102, 9898.

[101] S. Chen, R. W. Murray, J. Phys. Chem. B 1999, 103, 999.

[102] R. Guo, D. Genganopoulou, S. W. Feldberg, R. Donkers, R. W. Murray, Anal. Chem. 2005, 77, 2662.

[103] B. Su, H. H. Girault, J. Phys. Chem. B 2005 , 109, 11427; 23925

[104] B. Quinn, P. Liljeroth, V. Ruiz, T. Laaksonen, K. Kontturi, J. Am. Chem. Soc. 2003, 125, 6644.

[105] S. F. L. Mertens, K. Blech, A. S. Sologubenko, J. Mayer, U. Simon, T. Wandlowski, Electrochim. Acta 2009, 54, 5006.

[106] S. Chen, R. Pei, J. Am. Chem. Soc. 2001, 123, 10607.

[107] J. G. A. Dubois, J. W. Geritsen, G. Schmid, H. V. Kempen, Physica B 1996, 218, 262

[108] C. A. Niehuis, N. Oncel, J. Huiskens, H. J. W. Zandvliet, B. J. Ravoo, B. Poelsema, D. N. Reinhoudt, Small 2006, 21422.

[109] M. Homberger, U. Simon, Phil. Trans. R. Soc. A 2010, 368, 1405.

[110] T. Albrecht, S. F. L. Mertens, J. Ulstrup, J. Am. Chem. Soc. 2007, 129, 9162. 